\title{
Evaluation of evidence for the psychotic subtyping of post-traumatic stress disorder
}

\author{
Brandon A. Gaudiano and Mark Zimmerman
}

\begin{abstract}
Summary
Psychotic symptoms may occur in 15-64\% of individuals with post-traumatic stress disorder, suggesting that the syndrome could be subtyped in a similar fashion to mood disorders. In our study of 1800 psychiatric out-patients who completed comprehensive diagnostic interviews, the lifetime prevalence of psychotic symptoms in people with PTSD was 17\% (odds ratio $(\mathrm{OR})=3.48,95 \% \mathrm{Cl} 2.32-5.21)$. However, after excluding people with comorbid conditions also known to be
\end{abstract}

associated with psychotic symptoms this dropped to only $2.5 \%(\mathrm{OR})=0.60,95 \% \mathrm{Cl} 0.08-4.52)$. In contrast, rates of psychotic major depression did not change after excluding these same comorbidities. Our results do not support the official psychotic subtyping of PTSD.

\section{Declaration of interest}

None.
Although major depressive disorder and bipolar disorder have recognised psychotic subtypes, anxiety disorders with secondary psychotic features are not recognised in current psychiatric nosological systems. Nevertheless, researchers have long noted that psychotic symptoms frequently seem to co-occur in disorders such as post-traumatic stress disorder (PTSD). Braakman et al ${ }^{1}$ reviewed 24 studies and reported that psychotic symptoms in PTSD were present in as many as $15-64 \%$ of participants. Many authors have argued that PTSD should be psychotically subtyped in a manner similar to primary mood disorders and be included in future editions of the DSM. ${ }^{1,2}$ However, Braakman et al also noted that the studies they reviewed had many methodological limitations. Most earlier studies failed to thoroughly account for the confounding influence of the comorbid disorders, which themselves are known to be independently associated with psychotic symptoms (for example major depressive disorder). ${ }^{3}$ Furthermore, most earlier studies have not carefully differentiated the re-experiencing symptoms of PTSD from independent psychotic symptoms in their diagnostic and assessment procedures, leading to inflated rates. ${ }^{4}$ In the current study from the Rhode Island Methods to Improve Diagnostic Assessment and Services project, we investigated the lifetime prevalence rates of psychotic symptoms in individuals diagnosed with PTSD before and after we accounted for the effects of comorbid conditions with psychotic symptoms thus allowing us to examine support for the psychotic subtyping of PTSD.

\section{Method}

Participants included 1800 individuals presenting for treatment at a US psychiatry out-patient practice. The sample consisted of 1106 females $(61.4 \%)$ with a mean age of 37.7 (s.d.=12.6). The majority of the sample was White $(87.3 \%)$, followed by Hispanic (6.3\%), African American (4.1\%) and other ethnicities (2.4\%). Many participants were married $(40.8 \%)$ and over half of the sample $(53.7 \%)$ had a high-school degree or equivalent.

Individuals new to the clinic were offered the opportunity to have a more comprehensive evaluation as part of an Institutional Review Board-approved study, although they were not required to undergo this evaluation. Those who did and did not participate generally were comparable on self-report diagnostic questionnaires. ${ }^{5}$ The Structured Clinical Interview for DSM-IV ${ }^{6}$ and Structured Interview for DSM-IV Personality ${ }^{7}$ were used for Axis I and II diagnoses, respectively. Diagnosticians underwent extensive training to ensure reliability, including review of written cases, observation of at least 5 interviews, administration of 15 to 20 interviews under supervision and demonstration of exact or near-exact reliability with a senior diagnostician on 5 consecutive interviews. Diagnosticians then received ongoing supervision of interviews via a weekly case conference. Reliability was examined in 65 joint interviews: major depressive disorder $(\kappa=0.90)$, bipolar disorder $(\kappa=0.75)$, panic disorder $(\kappa=0.95)$, post-traumatic stress disorder $(\kappa=0.87)$, substance use disorders $(\kappa=0.64)$ and borderline personality disorder $(\kappa=1.0)$.

Diagnosticians were trained to carefully distinguish between psychotic symptoms versus the flashbacks and dissociative experiences often associated with PTSD. Psychotic major depressive disorder was diagnosed only when the perceptual disturbances were outside the realm of any trauma-related material. If the symptom was directly attributable to the actual traumatic event (for example hearing the voice of the perpetrator) then this was considered part of the PTSD syndrome. However, if the symptom was different from the traumatic event itself (for example hearing a disparaging voice that does not sound like the perpetrator), this was diagnosed as major depressive disorder with psychotic features if the experiences also occurred exclusively during a depressive episode.

\section{Results}

A total of 21\% $(n=378)$ had a lifetime PTSD diagnosis associated with the following traumas: sexual assault $(40.8 \%)$, non-sexual assault $(25.1 \%)$, witnessed death/violent assault (12.4\%), serious accident $(6.9 \%)$, military combat $(1.9 \%)$ and other $(9.8 \%)$. In participants with PTSD, $17.2 \%(n=65)$ had a lifetime history of psychotic symptoms, with $47.7 \%(n=31)$ reporting delusions and $72.3 \%(n=47)$ reporting hallucinations. Lifetime prevalence of psychotic symptoms in PTSD without major depressive disorder was $23.9 \%(n=38)$ compared with $4.6 \%(n=28)$ in major depressive disorder without PTSD, $12.3 \%(n=27)$ in PTSD with major depressive disorder and $10.0 \%(n=81)$ in all other diagnoses $\left(\chi^{2}=56.57\right.$, d.f. $\left.=3, P<0.001\right)$. The odds ratio for psychotic symptoms in PTSD without comorbid major depressive disorder was 3.48 (95\% CI 2.32-5.21). Prevalence of psychotic PTSD was recalculated after excluding comorbid conditions ${ }^{4}$ present in the sample in which psychotic symptoms also could be diagnosed according to DSM-IV: ${ }^{8}$ a psychotic disorder (for example schizophrenia), bipolar disorder, substance use disorder 


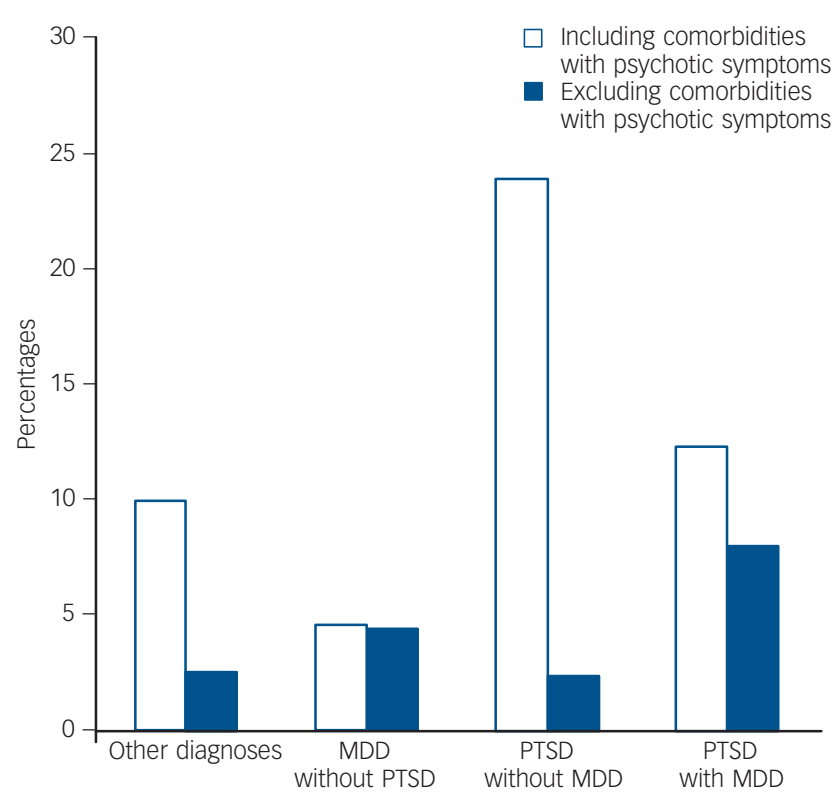

Fig. 1 Comparison of lifetime rates of psychotic symptoms by diagnostic category. MDD, major depressive disorder; PTSD, post-traumatic stress disorder.

or borderline personality disorder. After excluding these comorbidities, lifetime prevalence of psychotic symptoms in people with PTSD was $2.4 \% \quad(n=1)$ compared with $4.5 \%$ $(n=15)$ in major depressive disorder, $8 \%(n=7)$ in PTSD with major depressive disorder and 2.6\% $(n=10)$ in other diagnoses $\left(\chi^{2}=6.06\right.$, d.f. $\left.=3, P=0.109\right)$ (Fig. 1$)$. The odds ratio for psychotic symptoms in participants with PTSD excluding the above comorbidities (along with major depressive disorder) was 0.60 (95\% CI 0.08-4.52). When we restricted our analyses to people with current PTSD, lifetime rates of psychotic symptoms were $56 \%$. However, after excluding comorbid diagnoses associated with psychotic symptoms, $0 \%$ had psychotic symptoms.

\section{Discussion}

Consistent with previous research, $17 \%$ of the participants with PTSD reported a history of psychotic symptoms. Overall, people diagnosed with PTSD had nearly three and a half times the odds of experiencing psychotic symptoms. However, after excluding comorbid conditions with psychotic symptoms, rates of psychotic PTSD alone dropped to only $2.4 \%$ (1 participant). For an official psychotic subtype of PTSD to be considered valid, we would have expected a significant proportion of people with psychotic PTSD to remain. In contrast, exclusion of the same comorbid conditions did not affect the proportion of psychotic major depressive disorder in the sample $(4.6 \% v .4 .5 \%)$, suggesting that psychotic depression is not confounded by these comorbid diagnoses. Interestingly, individuals with comorbid PTSD and major depressive disorder appeared to have somewhat higher rates of psychotic symptoms (8\%), perhaps suggesting the synergistic effects of depression on PTSD and psychosis.

Sareen and colleagues ${ }^{3}$ reported that comorbid disorders did not account for the relationship between psychotic symptoms and PTSD using the National Comorbidity Survey database. However, these authors examined the effects of each comorbid disorder independently without careful differential diagnosis by a skilled interviewer. In other words, the effects of all the comorbid disorders combined that could be associated with psychotic symptoms were not controlled for in Sareen et al's analyses. ${ }^{3}$ Furthermore, the psychotic screen used in the original National Comorbidity Survey had poor validity and tended to overdiagnose symptoms. ${ }^{9}$ The current study findings suggest it is the cumulative effect of a number of different conditions that are comorbid with PTSD that appears to account for its strong association with psychotic symptoms.

Psychotic symptoms in PTSD have been reported to be particularly high in military veterans. ${ }^{10}$ Although there is no clear evidence that would predict differences in psychosis based on the type of Criterion A event, this could be investigated further in future studies in combat populations. In contrast, our sample was much more consistent with routine psychiatric practice settings, representing the majority of people with PTSD. Furthermore, we did not collect data on the timing of psychotic symptom onset as this is not recorded by the SCID. Thus, we were not able to consider temporal precedence, a topic that future research should continue to explore. Our findings suggest that PTSD in itself is not associated with psychotic symptoms after carefully differentiating trauma re-experiencing symptoms from psychotic symptoms and controlling for the effects of comorbid conditions also known to be associated with these symptoms.

Brandon A. Gaudiano, PhD, Department of Psychiatry \& Human Behavior, Alpert Medical School of Brown University, and Psychosocial Research Program, Butler Hospital, Providence; Mark Zimmerman, MD, Department of Psychiatry \& Human Behavior, Alpert Medical School of Brown University, and Department of Psychiatry, Rhode Island Hospital, Providence, Rhode Island, USA

Correspondence: Brandon A. Gaudiano, Butler Hospital, Psychosocial Research Program, 345 Blackstone Boulevard, Providence, Rhode Island 02906, USA. Email: Brandon_Gaudiano@brown.edu

First received 15 Feb 2010, final revision 8 July 2010, accepted 22 Jul 2010

\section{Funding}

The preparation of this manuscript was supported in part by a grant from the National Institute of Mental Health (MH076937) awarded to B.A.G.

\section{References}

1 Braakman MH, Kortmann FA, van den Brink W. Validity of 'post-traumatic stress disorder with secondary psychotic features': a review of the evidence. Acta Psychiatr Scand 2009; 119: 15-24.

2 Sautter FJ, Bissette G, Wiley J, Manguno-Mire G, Schoenbachler B, Myers L, et al. Corticotropin-releasing factor in posttraumatic stress disorder (PTSD) with secondary psychotic symptoms, nonpsychotic PTSD, and healthy control subjects. Biol Psychiatry 2003; 54: 1382-8.

3 Sareen J, Cox BJ, Goodwin RD, Asmundson JG. Co-occurrence of posttraumatic stress disorder with positive psychotic symptoms in a nationally representative sample. J Trauma Stress 2005; 18: 313-22.

4 Pierre JM. Hallucinations in nonpsychotic disorders: toward a differential diagnosis of "hearing voices". Harv Rev Psychiatry 2010; 18: 22-35.

5 Zimmerman M, Mattia Jl. The Psychiatric Diagnostic Screening Questionnaire: development, reliability and validity. Compr Psychiatry 2001; 42: 175-89.

6 First MB, Spitzer RL, Williams JBW, Gibbon M. Structured Clinical Interview for DSM-IV (SCID). American Psychiatric Association, 1997.

7 Pfohl B, Blum N, Zimmerman M. Structured Interview for DSM-IV Personality: SIDP-IV. American Psychiatric Association, 1997.

8 American Psychiatric Association. Diagnostic and Statistical Manual of Mental Disorder (4th edn) (DSM-IV). APA, 1994.

9 Kendler KS, Gallagher TJ, Abelson JM, Kessler RC. Lifetime prevalence, demographic risk factors, and diagnostic validity of nonaffective psychosis as assessed in a US community sample. The National Comorbidity Survey. Arch Gen Psychiatry 1996; 53: 1022-31.

10 David D, Kutcher GS, Jackson El, Mellman TA. Psychotic symptoms in combat-related posttraumatic stress disorder. J Clin Psychiatry 1999; 60: 29-32. 